\title{
Investigation of Mechanical Properties of Aluminum Composite (Al-7075/WC\& Fly-Ash) Fabricated by Stir Casting Process
}

\author{
A. Dhilipa \\ \{dlp.prod@psgtech.ac.in\} \\ aAssistant Professor, Department of Production Engineering, PSG College of Technology, \\ Coimbatore,
}

\begin{abstract}
This present investigation deals with the variation of mechanical properties of Al-7075 (WC and fly-ash) - a hybrid metal matrix composite prepared by using Al-7075 as matrix with Tungsten Carbide and fly ash as its primary and secondary reinforcements. The main objective of this work is to evaluate the effectof adding Tungsten Carbide (WC) and fly-ash to Al-7075 alloy by performing some mechanical tests. The volume fraction of reinforcements used was 2.5, 5.0 and 7.5 wt. \% of WC and fly-ash correspondingly. The technique used for fabrication of this hybrid compositewas stir casting and the reinforcements were added into the molten metal at $803^{\circ} \mathrm{C}$ (optimum). The composites fabricated were then machined according to ASTM dimensions as required for the mechanical tests. From the mechanical tests performed it was found that the hardness values and the toughness values increasedand the tensile strength decreased upon the addition of WC and fly-ash into the aluminium matrix. The improved values of hardness and toughness were because of uniform distribution and the decrease of tensile strength was due to the porosity which was clearly seen in the SEM image of the samples taken.
\end{abstract}

Keywords: Aluminium Matrix Composites (AMC's);Tungsten Carbide (WC); Fly-ash; Stir casting; SEM image.

\section{Introduction}

Metal matrix composites (MMCs) are a material combination that achieves the desired qualities. The development of numerous composite materials as a serious competitor to traditional engineering alloys has led to the development of numerous composite materials as a serious competitor to traditional engineering alloys of particular interest in the aerospace and defence industry, especially for Aluminium Metal Matrix Composites, in the last decade. Zinc is the main alloying element of Al-7075, which is an aluminium alloy.

MMC's having comparatively soft materials like aluminum, can be enhanced with its mechanical properties by adding predominantly hard materials like $\mathrm{B}_{4} \mathrm{C}[1]$, $\mathrm{TiC}$ [2], $\mathrm{SiC}$ [1, $3]$, WC $[4,6,7]$. However, ductility of MMC's decreases upon theaddition of such ceramic particles more than allowable percentage. The matrix material, reinforcement material, volume ofreinforcement material and the fabrication method can all be varied to achieverequired properties. Basavarajappa et al., have proven that aluminum alloy reinforced with $\mathrm{SiC}$ and fly ash have increased mechanical properties with increase in volume fraction. Aluminium metal 
matrix composites have unique properties such as high specific strength, good wear resistance, high strength to weight ratio, creep resistance, low density, high stiffness, low coefficient ofthermal expansion, corrosion resistance among others when compared with its counterpart. Various methods to manufacture metal matrix composites includes; solid state techniques, semi- solid state and liquid state techniques. Out of these techniques, liquid melt stirring process is quite advantageous because it is simple, economically viable, flexible, and capable of producing net shaped products and alsobetter matrix reinforcement bonding [5].But, problems associated with stir casting are its porosity along with poor wettability between matrix and reinforcement material.Strong bonds at the interface between matrix and reinforcement are required for good wetting. Poor wettability means matrix material cannot wet the reinforcement material, preventing homogeneity between both the materials, as a result of which reinforcement material float on the molten metal surface.

Mechanical stirring is the best solution to prevent such issue, but when stirring stops particles return to surface of melt. To prevent this, heat treatment of reinforcement before melting results in removal of oxides present within the reinforcement material. Therefore, good wetting is a most important criterionfor the generation of a satisfactory bond between particulate reinforcements and liquid aluminium metal matrix. During manufacturing composites using liquid processing techniques, to allow transfer and distribution of load from the matrix to the reinforcements without failure during mechanical loading pre-heating of reinforcements are necessary. These bonds may be formed by mutual dissolution of the particulates and matrix material.

To identify the research gap and recent trends in the fabrication of aluminium composites, few surveys on literatures related to the area of interest was made.

Ravi et al. ${ }^{[1]}$ performed characterization of aluminium (Al-6061) matrix composite reinforced with Boron Carbide $\left(\mathrm{B}_{4} \mathrm{C}\right)$ of size $25 \mu \mathrm{m}$ which was fabricated using stir casting technique. It was concluded that there was increase in tensile strength and hardness when there was an increase in percentage of $\mathrm{B}_{4} \mathrm{C}$ particles.

Lekatou et al. ${ }^{[2]}$ investigated the effect of having tungsten carbide (WC) and titanium carbide (TiC). A comparative study between these two composites fabricated using stir casting setup was made. It was inferred thatthe aluminium composite reinforced with tungsten carbide had better mechanical properties like hardness and comparatively low cost compared with Titanium carbide.

Subramanya Reddy et al. ${ }^{[3]}$ evaluated the mechanical properties of hybrid composite having Silicon Carbide $(\mathrm{SiC})$ and Boron Carbide $\left(\mathrm{B}_{4} \mathrm{C}\right)$ as reinforcements fabricated using stir casting process. As a result, the fabricated hybrid composite showed increased tensile strength, flexural rigidity and hardness than pure aluminium 6061.

Simon et al. ${ }^{[4]}$ conducted experiments on characterization of aluminium matrix composite reinforced with Tungsten Carbide (WC) using powder metallurgy process.It was deducted that,with the increase in percentages of WC nanoparticles, wear resistance and micro-hardness increased but the porosity increased.

Bhandare and Sonawane ${ }^{[5]}$ fabricated and performed characterizationof aluminium metal matrix composite using stir casting. The results obtained showed that the mechanical properties was enhanced only when total reinforcement was below $18 \%$ by weight percentage and also concluded that stir casting was simple, economical, and flexible than the other composite fabrication process.

Srikanth and Amarnath ${ }^{[6]}$ have determined the mechanical properties of Aluminium 6061 reinforced with tungsten carbide and fly-ash fabricated using stir casting setup. Tungsten carbide was taken in $1 \%, 2 \%, 3 \%$ followed by fly ash of $2 \%, 4 \%$, and $6 \%$ on mass fraction. 
The results indicated that the hardness and tensile strength increased with uniform distribution of WC and fly ash.

Arun B. Nallabelli et al. ${ }^{[7]}$ investigated the mechanical properties of Aluminium composite reinforced with $1.5 \%, 3.0 \%, 4.5 \%$ and $6.0 \%$ of Tungsten carbide to Al-7075. The obtained results indicated that with increase in addition of $\mathrm{WC}$ particles, micro-hardness and tensile strength was increased but the fracture toughness decreased with increase in composition of tungsten carbide.

Anil Kumar et al. ${ }^{[8]}$ found that the compressive andtensile strength followed by the hardness of the aluminium composites decreased with the increase in particle size of fly-ash. It was also found that when fly-ash was added as reinforcement, the voids formed due the stirring action were occupied by fly-ash thereby acting as a filler medium.

From the literatures related to aluminium composites fabricated using stir casting technique, it was noted that aluminium which was too soft was hardened by the use of ceramic particles. This was done to enhance the mechanical properties of aluminium and also to widen the scope of application of aluminium. So, Tungsten Carbide (WC) was chosen as a ceramic reinforcement and to limit the formation of pores during stir casting, fly-ash was chosen as the other reinforcement.

\section{Materials and Methods}

Aluminium 7075 has been chosen as the matrix for fabrication of aluminium matrix composites (AMC's). This aluminium 7075 grade was purchased from Coimbatore metal mart. Al-7075 is lighter in weight and has comparatively good corrosion resistance than other aluminium grade because of the presence of primary alloying element Zinc ( $\mathrm{Zn}$ ). The light weight makes it usable for manufacturing aircraft body parts.

\subsection{Compositional Analysis}

The chemical composition the aluminium alloy 7075 is shown in the table 1 below. This aluminium alloy was analysed using

\begin{tabular}{ccccccccccc}
\hline Element & Zn & Fe & Ti & Cu & Si & Pb & Mn & Mg & Cr & Al \\
\hline$\%$ & 5.48 & 0.24 & 0.042 & 1.42 & 0.094 & 0.0006 & 0.056 & 2.21 & 0.21 & 90.16 \\
\hline
\end{tabular}

Table-1 Composition of Al-7075 alloy

\subsection{Selection of Reinforcement Particles}

For this work, Tungsten Carbide (WC) and Fly-ash were chosen as reinforcements. WC was used to tailor the properties such as hardness, high temperature creep resistance and wear resistance and fly-ash was used to act as a filler medium during pores formation while stirring. The sizes of WC and fly-ash ranges from $110 \mu \mathrm{m}$ to $150 \mu \mathrm{m}$ and $70 \mu \mathrm{m}$ to $90 \mu \mathrm{m}$ respectively. The figure 1 and 2 below shows the Scanning Electron Micrograph (SEM) image of WC and fly-ash under optimum conditions respectively. 


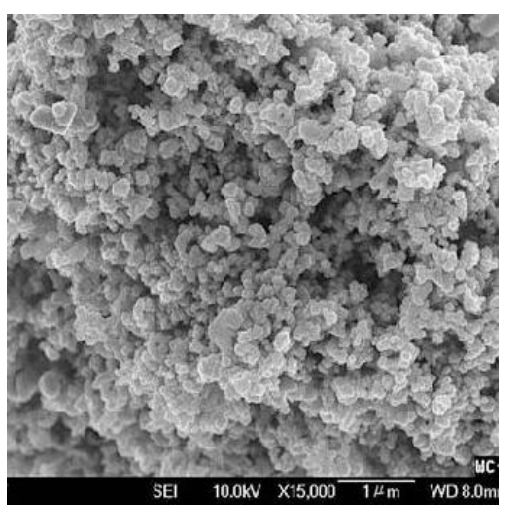

Fig.1: SEM image of WC

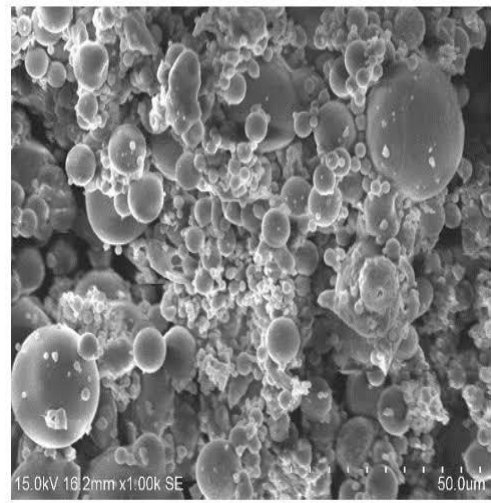

Fig.2: SEM image of Fly-Ash

\subsection{Composition of AMC's}

Totally 5 variety of compositions were fabricated by varying the weight percentages of WC and Fly-ash from $2.5 \%$ to $7.5 \%$ with a step of $2.5 \%$. The compositions of the composites fabricated are shown in table-2.

\begin{tabular}{cccc}
\hline Samples & $\begin{array}{c}\text { Wt. \% of } \\
\text { AA-7075 }\end{array}$ & $\begin{array}{c}\text { Wt. \% of } \\
\text { WC }\end{array}$ & $\begin{array}{c}\text { Wt. \% of } \\
\text { Fly-ash }\end{array}$ \\
\hline 1 & $100 \%$ & 0 & 0 \\
2 & $92.5 \%$ & 0 & $7.5 \%$ \\
3 & $92.5 \%$ & $7.5 \%$ & 0 \\
4 & $92.5 \%$ & $2.5 \%$ & $5.0 \%$ \\
5 & $92.5 \%$ & $5.0 \%$ & $2.5 \%$ \\
\hline
\end{tabular}

Table-2 Composition of AMC's

\subsection{Experimental Work}

Estimated amount ofaluminium 7075 was fed into the furnace and the temperature was increased to $803^{\circ} \mathrm{C}$ in order to melt the metal. Also, the reinforcements are mixed together and preheated to $800^{\circ} \mathrm{C}$ to remove any moisture content and also to increase the wettability between the two reinforcements. Once the aluminium is completely in molten state, the furnace was opened and the mixture of reinforcements was added into the molten metal 
accompanied by continuous stirring at 700rpm. This mixture was again heated beyond $800^{\circ} \mathrm{C}$ for 15 minutes. Then the molten mixture was cast into required dies to get the necessary specimens for the mechanical tests. The above procedure was followed to get the AMC's of different composition having wt. $\%-2.5 \%, 5.0 \%$ and $7.5 \%$ of $\mathrm{WC}$ and $2.5 \%, 5.0 \%$ and $7.5 \%$ of Fly-Ash. The stir casting experimental setup is shown in the figure 3.

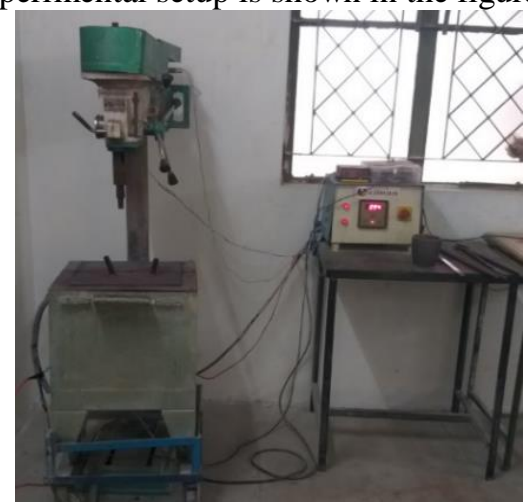

Fig.3: Stir casting setup

\subsection{Sample Preparation}

After the casting of molten aluminium into the split open die made of Cast Iron (C.I), the components were knocked out from their respective tensile, impact and billet dies as indicated in figures 4, 5 and 6. Each of the components was machined using CNC HTC, VMC and tool used was of the grade VMBT 04 having a nose radius of $0.4 \mathrm{~mm}$.

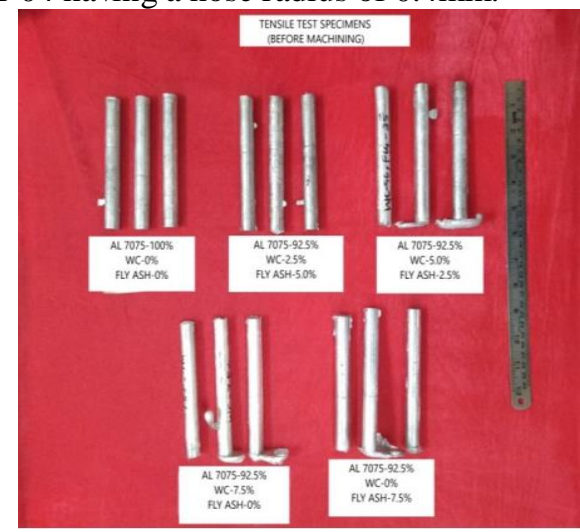

Fig.4: Tensile specimens after stir casting 


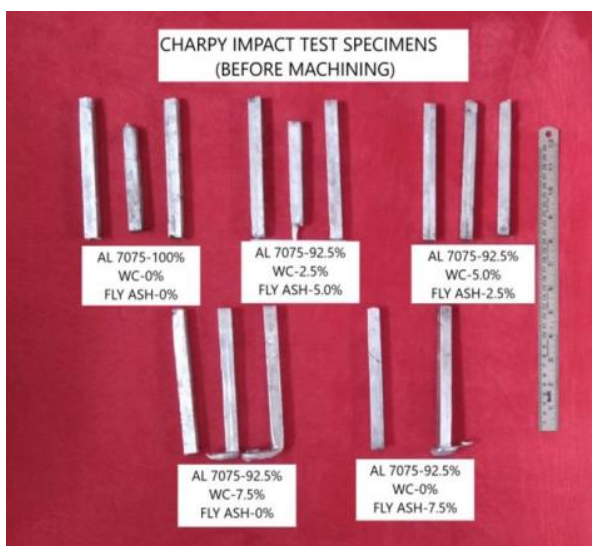

Fig.5: Impact specimens after stir casting

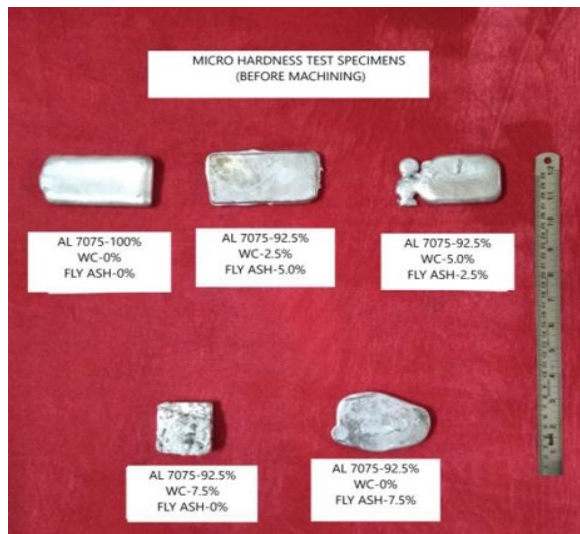

Fig.6: Micro-hardness specimens after stir casting

The dimensions followed were as per the ASTM for composites. For the tensile specimen ASTM E8M, Charpy impact test ASTM E23 and for hardness specimens, the dimensions as required by the material testing laboratory was used. The specimens that were machined andused for the tensile test, charpy impact test and micro-hardness test are clearly shown in figures 7,8 and 9 . 


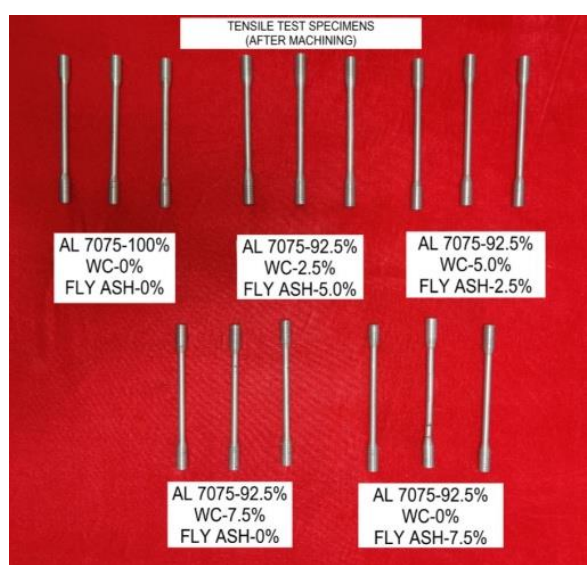

Fig.7: Tensile specimens after machining

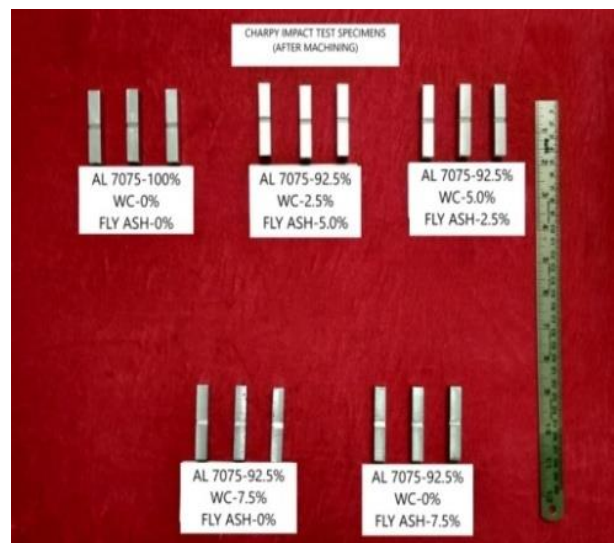

Fig.8: Impact specimens after machining

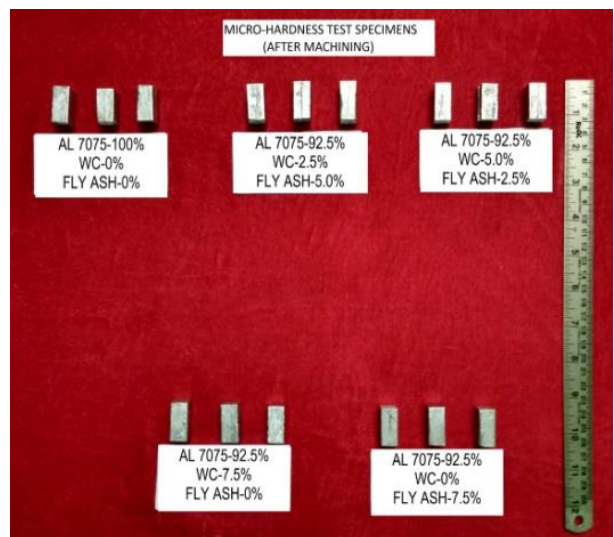

Fig.9: Micro-hardness specimens after machining 


\section{Testing of Mechanical Properties}

The fabricated specimens were given for mechanical tests like tensile test, Charpy impact test and micro-hardness test along with this the SEM images were also captured to identify the dispersion of reinforcements into aluminium matrix.

\subsection{Tensile Test}

In order to analyze the tensile properties of the composites fabricated using Al-7075, tensile test is carried out on a computerized Universal Tensile testing machine (UTM) for the specimens machined according to ASTM E8M standard for composites. Properties such as tensile strength, yield stress, percentage of elongation and reduction area were analyzed.

\subsection{Charpy Impact Test}

Impact test was carried out to evaluate the fracture toughness of the composite. Out of various impact tests available, charpy test was chosen for the specimens machined according to ASTM E23 standard for composites.

\subsection{Micro-hardness Test}

Hardness test was carried out to identify whether the fabricated composites shows any resistance to indentation. Micro-hardness was chosen especially because of the smaller size of specimen and also to prevent any breakage of specimen. The load applied was $1.962 \mathrm{~N}$ for duration of 30 seconds.

\subsection{Microstructural Study}

Microstructural study was done to identify the dispersion of WC and fly-ash into the aluminium 7075 matrix. This study was made possible by the use of micrograph images captured using Scanning Electron Microscope (SEM) equipped with Energy Dispersive X-ray Spectroscopy (EDS)

\section{Results and Discussions}

\subsection{Tensile Test}

The tensile test results are shown in table-3. This test revealed that, the ultimate tensile strength (UTS) increased with the increase in wt. \% of fly-ash but when there wasan increase in wt. \% of WC, the UTS decreased. The UTS value increased beyond the value of pure Al7075 when fly-ash alone was added without WC, and it decreased when WC was alone added without fly-ash as indicated in the trend shown in figure 10.

\begin{tabular}{ccccc}
\hline Samples & $\begin{array}{c}\text { Ultimate Tensile Strength } \\
(\mathbf{M P a})\end{array}$ & $\begin{array}{c}\text { Yield Stress } \\
(\mathbf{M P a})\end{array}$ & $\begin{array}{c}\text { Elongation } \\
(\boldsymbol{\%})\end{array}$ & $\begin{array}{c}\text { Reduction Area } \\
(\boldsymbol{\%})\end{array}$ \\
\hline 1 & 135.34 & 120.83 & 6.29 & 30.93 \\
2 & 171.647 & 150.60 & 4.42 & 29.76 \\
3 & 135.88 & 114.67 & 6.42 & 25.95 \\
4 & 160.51 & 151.63 & 5.87 & 26.32 \\
5 & 156.55 & 143.08 & 6.71 & 27.37 \\
\hline
\end{tabular}


Similarly in the case of Yield stress, the trend was same as that of UTS and so the values of yield stress increased with the increase in wt. \% of fly-ash and decreased with the increase in wt. \% of WC as shown in figure 10 . This was because, as fly-ash acted as a filler medium, the samples 2 and 4 having more wt. \% of fly-ash had less porosity thereby contributing to a major increase in values of UTS and yield stress. On the other hand, there was only a slight increase in values of UTS and yield stress for samples 3 and 4 was due to increased wt. \% of WC and those samples were found with significant porosityeither due to minimal presence or complete absence of fly-ash. However, as a whole, the composite fabricated showed increase in UTS and yield stress on comparison with pure Al-7075 fabricated by stir casting.

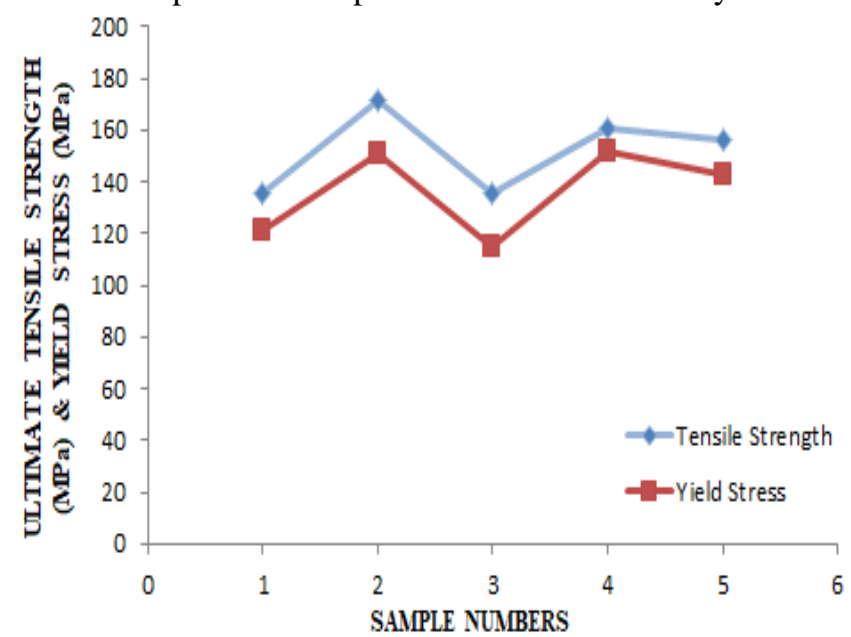

Fig.10: Plot showing the variation of UTS and Yield Stress with respect to different compositions

\subsection{Impact Test}

The impact test was carried out to analyze the fracture toughness of composites manufactured under the application sudden shock or impact loads. This experiment was carried out for 15 samples and the toughness values were tabulated as shown in table- 4 . Mean values for each sample were calculated and was used for inferences. Variation of toughness values between samples are shown in the figure 11 .

\begin{tabular}{ccc}
\hline Samples & $\begin{array}{c}\text { Fracture } \\
\text { Toughness } \\
(\mathbf{J})\end{array}$ & $\begin{array}{c}\text { Average } \\
(\mathbf{J})\end{array}$ \\
\hline \multirow{2}{*}{1} & 2 & \\
& 2 & 2 \\
& 2 & \\
2 & 1.8 & 1.93 \\
& 2 & \\
& 2 & \\
3 & 2.1 & \\
& 2.1 & 2.23 \\
4 & 2.5 & \\
& 2.3 & 1.9 \\
\hline
\end{tabular}




\begin{tabular}{lll}
\hline & 1.2 & \\
5 & 2.8 & \\
5 & 2.9 & 3.07 \\
& 3.5 & \\
\hline
\end{tabular}

Table-4 Charpy Impact Test Result

From the table 4, it was observed that, the sample 5 of composition having $5.0 \%$ of WC and $2.5 \%$ of fly-ash and remaining $92.5 \%$ of AA-7075 showed the maximum fracture toughness. Also, whenever the wt. \% of fly-ash was less, the toughness values increased (sample 3 and 5) and when wt. \% of fly-ash was more, the toughness values decreased (sample 2 and 4). The increase was because of uniformhomogeneous distribution of WC, flyash into the metal matrix and in addition to that, those samples werefound with less porosity. Also, as the reinforcements were pre-heated, the wettability between them was increased thereby forming a strong bond increasing the toughness. The variation of toughness values with respect to different samples fabricated is shown as a trend in the figure 11 below.

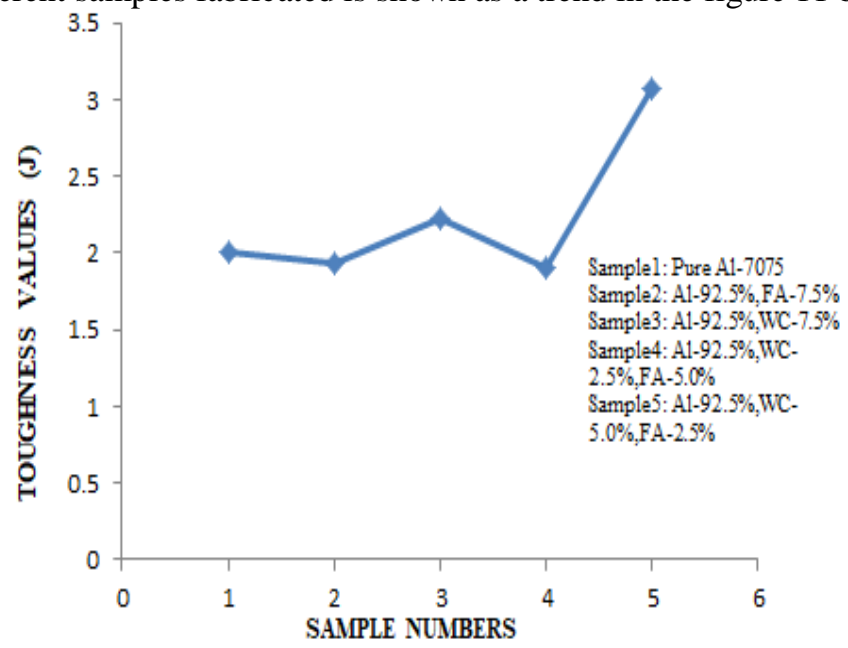

Fig.11: Plot indicating variation of toughness values with respect to different

\subsection{Micro-hardness Test}

compositions

The test results of micro-hardness are tabulated in table-5. This micro-hardness test was done to deduce the resistance to penetration of various composite samples fabricated. A micro load of $1.962 \mathrm{~N}$ was applied for time duration of 30seconds and the micro-hardness values were attained. As the sizes of the samples were small and they could break at any instance, the load was given on a micro scale. The type of hardness test used was Vickers micro-hardness test using a diamond indenter.

\begin{tabular}{ccc}
\hline Samples & $\begin{array}{c}\text { Micro-Hardness } \\
(\text { VHN) }\end{array}$ & $\begin{array}{c}\text { Average } \\
\text { (VHN) }\end{array}$ \\
\hline \multirow{2}{*}{1} & 101 & \\
& 100 & 103 \\
2 & 108 & 96 \\
\hline
\end{tabular}




\begin{tabular}{ccc}
\hline & 113 & \\
& 93 & \\
3 & 95 & 96.6 \\
& 107 & \\
& 88 & \\
4 & 92 & 92.3 \\
& 85 & \\
& 96 & 89.3 \\
5 & 82 & \\
\hline
\end{tabular}

Table-5 Micro-Hardness Test Result

From table 4 and the figure 12 below, it was inferred that sample 1 having pure Al-7075 alloy showed high micro-hardenss among all other compositions. It was noted that, whenever both reinforcements were used (samples 4 and 5), the micro-hardness values dropped to larger scale. And when either one of the reinforcement (WC or Fly-ash) was used (samples 2 and 3), there was not increase in micro-hardness, rather it dropped to a smaller extent. Whatever maybe the composition, the results was downward sloping. When two reinforcements were used there wasn't a uniform distribution in the Aluminium phase and when one of the reinforcement was used, the distribution was uniform to some extent. The increase or decrease of micro-hardness values was greatly influenced by the rapid solidifications in these alloys which results in micro segregation of $\mathrm{Mg}-\mathrm{Zn}$ phases that reduces hardening potential.

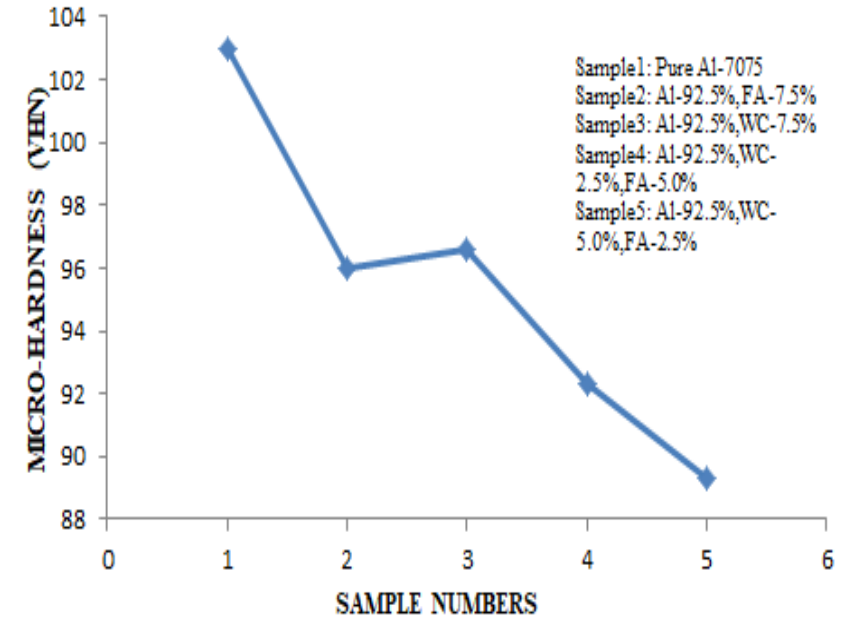

Fig.12: Plot indicating variation of Micro-hardness valueswith respect to different compositions

\subsection{Microstructural study}

The microstructural study was performed on the 5 samples fabricated in order to identify the particle distribution of reinforcements into the aluminium7075 matrix. This study was conducted from the micrograph images captured using a Scanning Electron Microscope (SEM) having Energy Dispersive X-ray Spectroscopy (EDS). 


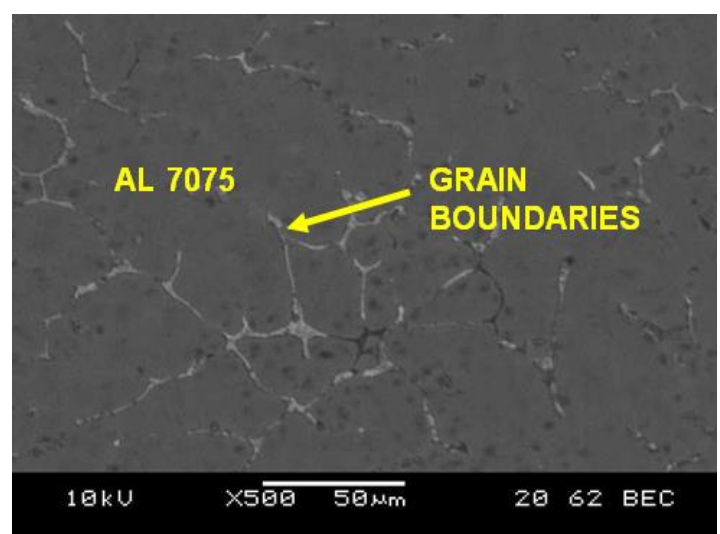

Fig.13: SEM image of sample 1 (Pure Al-7075)

The figure 13 depicts the SEM of pure Al-7075 alloy fabricated by stir casting. It was observed that grain boundaries were formed along the aluminium phases. Some amount of impurities were also found along the samples which may be due todifferential cooling of molten aluminium 7075 alloy while solidification. Apart from these, the numbers of pores found on the sample was less.

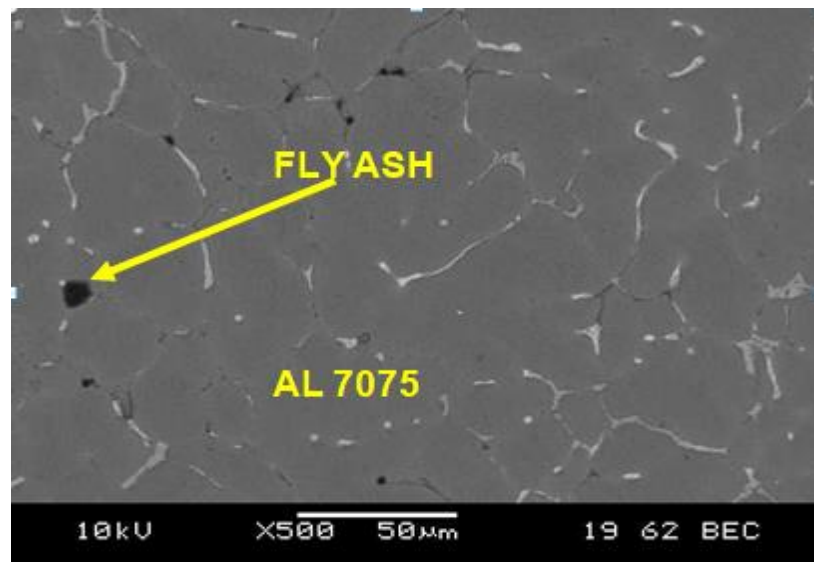

Fig.14: SEM image of sample 2 (Al-7075 92.5\%, FA-7.5\%, WC-0\%)

SEM image of sample 2 having $7.5 \%$ of fly-ash is shown in figure 14 . From this image, it was revealed that the fly-ash was found along the pores formed while solidification. This sample has high Ultimate Tensile Strength (UTS) and Yield Stress (shown in figure 11) due to the minimal amount of pores found on the sample and the aluminium phases were easily wetted by fly-ash. 


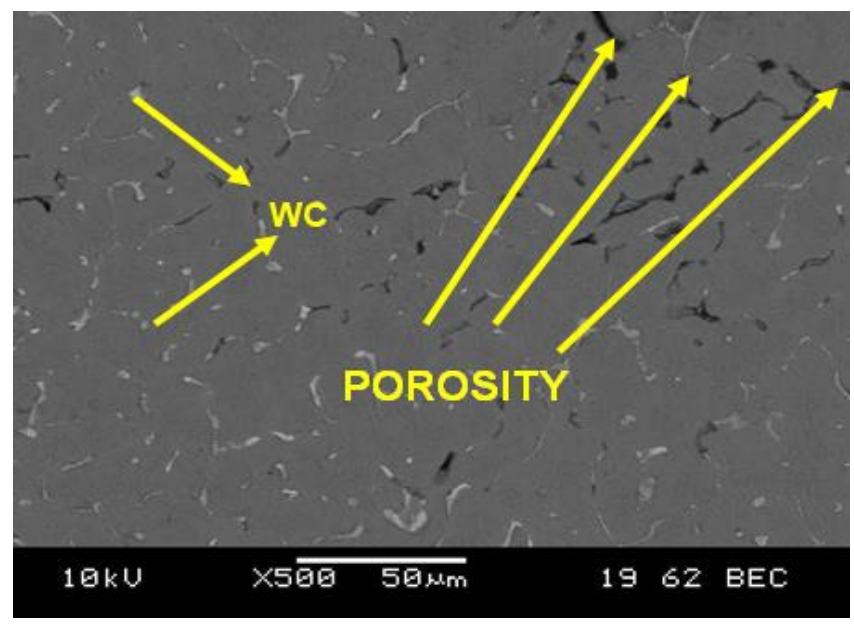

Fig.15: SEM image of sample 3 (Al-7075 92.5\%, FA-0\%, WC-7.5\%)

The figure 15 shows the SEM image of sample 3 fabricated by stir casting technique. Only Tungsten carbide (WC) of $7.5 \%$ to the total weight percentage is mixed into pure $\mathrm{Al}$ 7075. Due to the absence of fly-ash (filler medium), this sample was found with high amount of porosity due to which the fracture toughness values decreased beyond the toughness value of pure Al-7075 (shown in table-4).

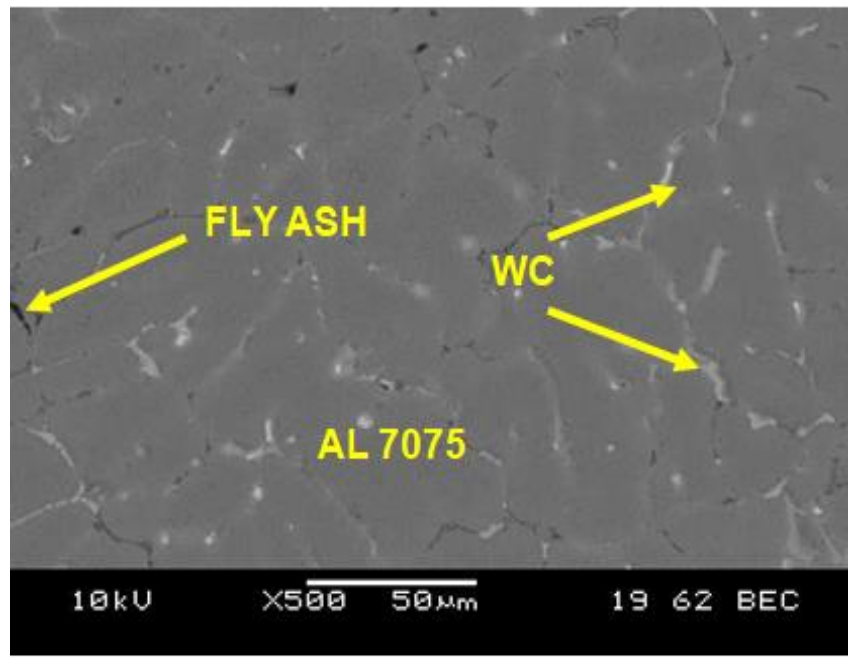

Fig.16: SEM image of sample 4 (Al-7075 92.5\%, FA-2.5\%, WC-5.0\%)

The SEM image of sample 4 is pictured in figure 16. From this image it can be inferred that, WC and fly-ash was uniformly distributed into the aluminium matrix. After the sample 2, this sample 4 showed high Ultimate Tensile Strength (UTS) and Yield Stress (shown in figure 11). Also, this sample 4 has low fracture toughness (shown in table-4). 


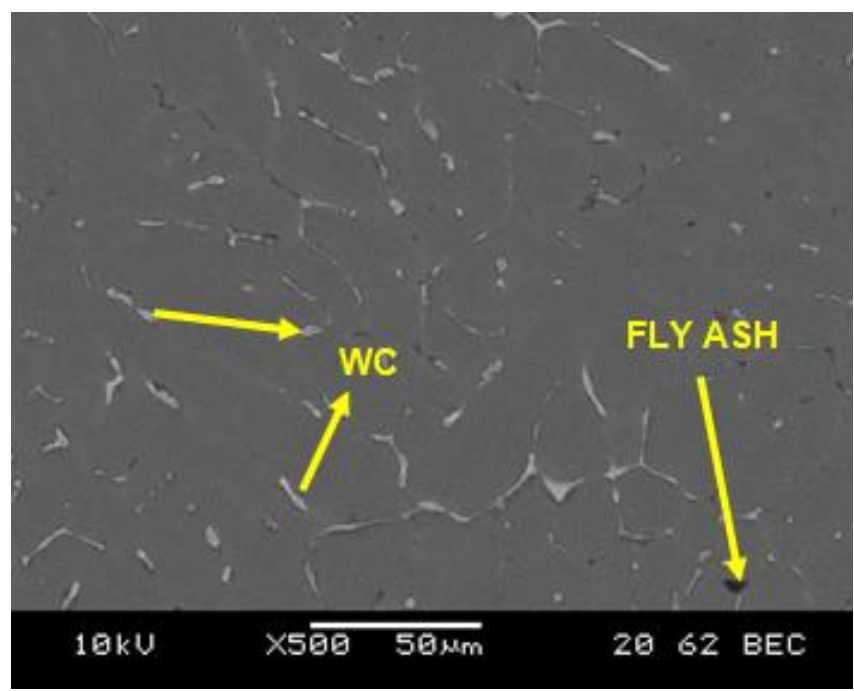

Fig.17: SEM image of sample 5 (Al-7075 92.5\%, FA-5\%, WC-2.5\%)

The figure 17 depicts the SEM image of sample 5 fabricated by stir casting. This sample has a mixture of fly-ash and tungsten carbide adding up to $7.5 \%$ to the total weight percentage of Al-7075 alloy. WC was found along the grain boundaries of aluminium phases thereby eliminating any discontinuity in the grain boundary formation. As fly-ash was used, the number of pores formed was limited. Due to this combined effect, this sample showed high fracture toughness (shown in table-4).

\section{Conclusions}

From the mechanicals tests performed, the results obtained are as follows;

(i) The ultimate tensile strength and yield stress values were found to be increased for composites having higher weight percentages of fly-ash.

(ii) The impact toughness values increased $37 \%$ for Sample 3 and decreased approximately $13 \%$ for other samples.

(iii) The micro-hardness values started decreasing on the addition of reinforcements. Sample 3 showed the least micro-hardness of $89.3 \mathrm{VHN}$.

(iv) From SEM images, it was concluded that Tungsten Carbide particles occupied the grain boundaries therefore the discontinuous grain boundaries were removed.

(v) Also, Fly-ash acted as filler material thereby decreasing the amount of pores formed while stir casting.

\section{References}

[1] B. Ravi, B. Balu Naik, J. Udaya Prakash "Characterization of aluminium matrix composites (AA 6061 / B4C) fabricated by stir casting technique", International journal of material processing and characterization, Volume-4, pp. 2984-2990, 2015. 
[2] A. Lekatou, A.E. Karantzalis, A. Evenglou, "Aluminium reinforced with TiC and WC particles and aluminide particles: Microstructure, wear and corrosion behaviour”, 2014.

[3] P. Subramanya Reeddy, R. Kesavan, B. Vijaya Ramnatth" Investigation of mechanical properties of aluminium 6061 - silicon carbide, boron carbide meal matrix composites", pp. 495-502, January 2017.

[4] A. Simon, D. lipuz, P. Balini, G. Kaptay, G. Gergely, A. Sfikas, A. Lekatou, A. Karantzalis, Z. Gacsi, "Microstructure and mechanical properties of Al-WC composites", International journal of metallurgy and materials, Volume-60, pp. 388-393, 2015.

[5] RajeshkumarGangaramBhandare,ParhuramM.Sonawane"Preparation of Aluminium Matrix Composite by Using Stir Casting Method",International Journal of Engineering and advanced Technology(IJEAT) ISSN:2249-8958,Volume-3.Issue-2,December2013.

[6] B.G. Srikanth, G. Amarnath," Characterization of aluminium reinforced with tungsten carbide particulate and fly ash metal matrix composites", International journal of engineering research and technology, Volume-4, pp. 623-327, 2015.

[7] ArunBabuNallabelli, BaluNaikBanoth, B. Ravi, G. Raja Kumar, "Evaluation of mechanical properties of Al 7075 reinforced with WC metal matrix composites", International journal of advanced information science and technology, Volume-6, pp. 213-217, 2017.

[8] Anilkumar, H.S. Hebbar and K.S. Ravishankar, "Mechanical Properties of Fly Ah Reinforced Aluminium Alloy (A16061) Composites"International Journal of Mechanical and Materials Engineering (IJMME), Vol.6, No.1, pp. 41-45, 2011.

[9] D. S. Vijayan, A. Mohan, J. J. Daniel, V. Gokulnath, B. Saravanan, and P. D. Kumar, "Experimental Investigation on the Ecofriendly External Wrapping of Glass Fiber Reinforced Polymer in Concrete Columns," vol. 2021, 2021. 